\title{
SUFFICIENT CONDITIONS FOR THE EXISTENCE OF A CENTER IN POLYNOMIAL SYSTEMS OF ARBITRARY DEGREE
}

\author{
H. Giacomini and M. Ndiaye
}

\begin{abstract}
In this paper, we consider polynomial systems of the form $\dot{x}=$ $y+P(x, y), \dot{y}=-x+Q(x, y)$, where $P$ and $Q$ are polynomials of degree $n$ wihout linear part.

For the case $n=3$, we have found new sufficient conditions for a center at the origin, by proposing a first integral linear in certain coefficient of the system. The resulting first integral is in the general case of Darboux type.

By induction, we have been able to generalize these results for polynomial systems of arbitrary degree.
\end{abstract}

\section{Introduction}

In this paper, we consider systems of differential equations

$$
\begin{aligned}
& \dot{x}=P(x, y), \\
& \dot{y}=Q(x, y),
\end{aligned}
$$

in which $P(x, y)$ and $Q(x, y)$ are polynomials of the form

$$
\begin{aligned}
& P(x, y)=y+\sum_{j=2}^{n} \sum_{i=0}^{j} a_{i j-i} x^{i} y^{j-i} \\
& Q(x, y)=-x-\sum_{j=2}^{n} \sum_{i=0}^{j} b_{i j-i} x^{i} y^{j-i}
\end{aligned}
$$

and we seek for sufficient conditions under which the origin is a center i.e., a critical point in a neighbourhood of which all orbits are closed. The problem of the center is an important one in the theory of plane polynomial vector fields. It plays an important role in the second part 
of Hilbert's $16^{\text {th }}$ problem, which asks for the maximum number of limit cycles that a vector field given by polynomials of degree $n$ should have.

The necessary and sufficient conditions for a center are known for very few classes of systems. There are well-known conditions for quadratic systems [1], [2], [3] (and references therein) and for systems in which $P(x, y)$ and $Q(x, y)$ are cubic polynomials without quadratic terms [4], [5]. Conditions have been obtained for other classes of cubic systems. For instance, in [6] and [7], Kukles studied systems of the form:

$$
\begin{aligned}
& \dot{x}=y, \\
& \dot{y}=-x+a_{1} x^{2}+a_{2} x y+a_{3} y^{2}+a_{4} x^{3}+a_{5} x^{2} y+a_{6} x y^{2}+a_{7} y^{3},
\end{aligned}
$$

and claimed to have found necessary and sufficient conditions under which the origin is a center. However, Jin and Wang showed in [8] that Kukles'conditions are only sufficient but not necessary, by giving explicitly another condition which is not covered by the Kukles'ones (see also [9]). C. J. Christopher and N. G. Lloyd showed in [10] that Kukles'conditions are necessary only in the case where $a_{7}=0$.

More recently, important results have been obtained about the problem of the center for cubic polynomial vector fields [11], [12].

For polynomial systems of arbitrary degree, there are three classes for which sufficient conditions for the existence of the center are known: a family of homogeneous polynomial vector fields [13], reversible system [14], the Hamiltonian case. For this case, the constant of motion (the Hamiltonian) is linear in all the coefficients of the polynomials $P(x, y)$ and $Q(x, y)$.

In the first section of this paper we analyse cubic systems. We propose a constant of motion which is linear in the coefficient of $y^{2}$ in the polynomial $P(x, y)$. The reason of this choice comes from the analysis of the form of the constant of motions for the quadratic systems [1], [2]. For one of integrability conditions, the first integral is linear in the coefficient $a_{02}$. For cubic system, by proposing a first integral linear in $a_{02}$, we have found several new sets of sufficient conditions for a center. The corresponding first integrals are in general case of Darboux type [15], $[\mathbf{1 6}],[\mathbf{1 7}],[\mathbf{1 8}]$. We have also proposed first integrals that are linear in the other coefficients of the system but we have not found new integrability conditions. Let us remark that the first integrals of Darboux type that we have found are not contains in the Sokulsky's list [12]. We verify that this associated constant of motion is also linear in the coefficients of $y$ and $y^{3}$ (by symmetry, analogous constants of motion exist that are linear in the coefficients of $x, x^{2}$ and $x^{3}$ of the polynomial $\left.Q(x, y)\right)$. It is natural to think that this result is valid for polynomial systems of 
an arbitrary degree. In order to check this hypothesis, we perform the calculations for polynomials $P(x, y)$ and $Q(x, y)$ of degree $4 \leq n \leq 8$. In all these cases, we find that the constant of motion is linear in the coefficients of $y^{j}$ of $P(x, y)$, with $1 \leq j \leq n$.

The analysis of the integrability conditions and the explicit expressions of constants of motion will enable us to conjecture a general result, valid for an arbitrary degree $n$.

The conjectured expressions for the integrability conditions and constants of motion reduce to the correct results for $2 \leq n \leq 8$.

These results, valid for arbitrary $n$, are given in Section 2 .

\section{Case $n=3$}

We consider the system:

$$
\begin{aligned}
& \dot{x}=y+\sum_{j=2}^{3} \sum_{i=0}^{j} a_{i j-i} x^{i} y^{j-i}, \\
& \dot{y}=-x-\sum_{j=2}^{3} \sum_{i=0}^{j} b_{i j-i} x^{i} y^{j-i} .
\end{aligned}
$$

We propose a constant of motion $f(x, y)$ linear in the coefficient $a_{02}$ :

$$
f(x, y)=f_{1}(x, y) a_{02}+f_{2}(x, y)
$$

where $f_{1}(x, y)$ and $f_{2}(x, y)$ are independent of $a_{02}$.

Taking the rate of change of $f(x, y)$ along a trajectory of $\left(S_{1}\right)$, we obtain

$$
\begin{aligned}
\dot{f}(x, y)= & \left(\frac{\partial f_{1}(x, y)}{\partial x} a_{02}+\frac{\partial f_{2}(x, y)}{\partial x}\right) P(x, y) \\
& +\left(\frac{\partial f_{1}(x, y)}{\partial y} a_{02}+\frac{\partial f_{2}(x, y)}{\partial y}\right) Q(x, y)
\end{aligned}
$$

If $f(x, y)$ is a constant of motion, $\dot{f}(x, y)$ must be identically zero for arbitrary values of $a_{02}$. In consequence, we obtain the following conditions for the functions $f_{1}(x, y)$ and $f_{2}(x, y)$ :

$$
\begin{array}{r}
\frac{\partial f_{1}(x, y)}{\partial x}=0 \\
Q(x, y) \frac{\partial f_{1}(x, y)}{\partial y}+y^{2} \frac{\partial f_{2}(x, y)}{\partial x}=0 \\
C(x, y) \frac{\partial f_{2}(x, y)}{\partial x}+Q(x, y) \frac{\partial f_{2}(x, y)}{\partial y}=0
\end{array}
$$


which imply

$$
\begin{aligned}
f_{1}(x, y) & =f_{1}(y) \\
\frac{\partial f_{2}(x, y)}{\partial x} & =-\frac{Q(x, y)}{y^{2}} \frac{\partial f_{1}(y)}{\partial y} \\
\frac{\partial f_{2}(x, y)}{\partial y} & =\frac{C(x, y)}{y^{2}} \frac{\partial f_{1}(y)}{\partial y}
\end{aligned}
$$

where

$$
C(x, y)=y+a_{20} x^{2}+a_{11} x y+a_{30} x^{3}+a_{21} x^{2} y+a_{12} x y^{2}+a_{03} y^{3} .
$$

By imposing the condition

$$
\frac{\partial^{2} f_{2}(x, y)}{\partial x \partial y}=\frac{\partial^{2} f_{2}(x, y)}{\partial y \partial x} .
$$

We obtain from $(\alpha)$ and $(\beta)$ :

$$
\begin{gathered}
\left(-a_{11} y^{3}+\left(-a_{12}+b_{03}\right) y^{4}\right) \frac{\partial f_{1}(y)}{\partial y}+\left(b_{02} y^{4}+b_{03} y^{5}\right) \frac{\partial^{2} f_{1}(y)}{\partial y^{2}} \\
+\left(\left(-2 y+\left(-2 a_{20}-b_{11}\right) y^{2}-2 a_{21} y^{3}\right) \frac{\partial f_{1}(y)}{\partial y}+\left(y^{2}+b_{11} y^{3}+b_{12} y^{4}\right) \frac{\partial^{2} f_{1}(y)}{\partial y^{2}}\right) x \\
+\left(\left(-2 b_{20} y+\left(-3 a_{30}-b_{21}\right) y^{2}\right) \frac{\partial f_{1}(y)}{\partial y}+\left(b_{20} y^{2}+b_{21} y^{3}\right) \frac{\partial^{2} f_{1}(y)}{\partial y^{2}}\right) x^{2} \\
+\left(\left(-2 y \frac{\partial f_{1}(y)}{\partial y}+y^{2} \frac{\partial^{2} f_{1}(y)}{\partial y^{2}}\right) x^{3}\right) b_{30}=0 .
\end{gathered}
$$

As this condition must be satisfied for arbitrary values of $x$ and $y$, and $f_{1}(y)$ is a function only of $y$, we have the following equations for the function $f_{1}(y)$ :

$\left(S_{2}\right)$

(1) $\left(-a_{11}+\left(-a_{12}+b_{03}\right) y\right) \frac{\partial f_{1}(y)}{\partial y}+\left(b_{02} y+b_{03} y^{2}\right) \frac{\partial^{2} f_{1}(y)}{\partial y^{2}}=0$

(2) $\left(-2+\left(-2 a_{20}-b_{11}\right) y-2 a_{21} y^{2}\right) \frac{\partial f_{1}(y)}{\partial y}+\left(y+b_{11} y^{2}+b_{12} y^{3}\right) \frac{\partial^{2} f_{1}(y)}{\partial y^{2}}=0$

(3) $\left(-2 b_{20}+\left(-3 a_{30}-b_{21}\right) y\right) \frac{\partial f_{1}(y)}{\partial y}+\left(b_{20} y+b_{21} y^{2}\right) \frac{\partial^{2} f_{1}(y)}{\partial y^{2}}=0$,

(4) $-2 \frac{\partial f_{1}(y)}{\partial y}+y \frac{\partial^{2} f_{1}(y)}{\partial y^{2}}=0$, or

(5) $b_{30}=0$. 
Then we must consider two different cases:

1) Equations (1), (2), (3) and (4) are satisfied:

These equations are compatible if and only if the following conditions holds

$-b_{21}+3 a_{30}=-b_{11}+2 a_{20}=-b_{12}+a_{21}=-2 b_{02}+a_{11}=-3 b_{03}+a_{12}=0$.

These conditions insure that System $\left(S_{1}\right)$ is Hamiltonian. Therefore, in order to obtain new results we must consider condition (1), (2), (3) and (5).

2) Equations (1), (2), (3) and (5) are satisfied.

The compatibility relation between (1) and (2) gives:

$$
\begin{aligned}
\left(-2 b_{02}\right. & \left.+a_{11}\right) y+\left(\left(-b_{11}-2 a_{20}\right) b_{02}-3 b_{03}+a_{12}+a_{11} b_{11}\right) y^{2} \\
+\left(-2 b_{11} b_{03}-2 a_{20} b_{03}-\right. & \left.2 a_{21} b_{02}+a_{12} b_{11}+a_{11} b_{12}\right) y^{3} \\
& +\left(-2 a_{21} b_{03}-b_{03} b_{12}+a_{12} b_{12}\right) y^{4}=0
\end{aligned}
$$

for arbitrary values of $y$.

The compatibility condition between (1)-(2) and (2)-(3) gives, respectively

$$
\begin{aligned}
\left(\left(-2 b_{02}+a_{11}\right) b_{20}\right) y+\left(-b_{21} b_{02}\right. & \left.-3 a_{30} b_{02}-3 b_{20} b_{03}+b_{20} a_{12}+b_{21} a_{11}\right) y^{2} \\
+ & \left(-2 b_{21} b_{03}-3 a_{30} b_{03}+b_{21} a_{12}\right) y^{3}=0 \\
\left(3 a_{30}-2 a_{20} b_{20}+b_{11} b_{20}-b_{21}\right) y^{2} & +\left(3 a_{30} b_{11}-2 a_{21} b_{20}+2 b_{12} b_{20}-2 a_{20} b_{21}\right) y^{3} \\
+ & \left(3 a_{30} b_{12}-2 a_{21} b_{21}+b_{12} b_{21}\right) y^{4}=0
\end{aligned}
$$

As these conditions must be satisfied for arbitrary values of $y$, we have

$$
\begin{aligned}
b_{30} & =0, \\
-2 b_{02}+a_{11} & =0, \\
-2 b_{11} b_{03}-2 a_{20} b_{03}-2 a_{21} b_{02}+a_{12} b_{11}+a_{11} b_{12} & =0 \\
-b_{11} b_{02}-2 a_{20} b_{02}-3 b_{03}+a_{12}+a_{11} b_{11} & =0 \\
-2 a_{21} b_{03}-b_{03} b_{12}+a_{12} b_{12} & =0 \\
-b_{21} b_{02}-3 a_{30} b_{02}-3 b_{20} b_{03}+b_{20} a_{12}+b_{21} a_{11} & =0 \\
-2 b_{21} b_{03}-3 a_{30} b_{03}+b_{21} a_{12} & =0 \\
3 a_{30}-2 a_{20} b_{20}+b_{11} b_{20}-b_{21} & =0 \\
3 a_{30} b_{11}-2 a_{21} b_{20}+2 b_{12} b_{20}-2 a_{20} b_{21} & =0 \\
3 a_{30} b_{12}-2 a_{21} b_{21}+b_{12} b_{21} & =0 .
\end{aligned}
$$


If we assume that

$$
\left(3 a_{30}+b_{21}\right)\left(3 a_{30}+2 b_{21}\right) \neq 0, \quad b_{20} \neq 0,
$$

the general solution of the above system of equations is

$$
\begin{aligned}
& b_{11}=\frac{2 a_{21} b_{21}}{3 a_{30}+b_{21}}+\frac{b_{21}}{b_{20}}, \quad b_{02}=\frac{a_{12} b_{20}}{3 a_{30}+2 b_{21}}, \quad b_{12}=\frac{2 a_{21} b_{21}}{3 a_{30}+b_{21}}, \\
& b_{03}=\frac{a_{12} b_{21}}{3 a_{30}+2 b_{21}}, \quad a_{20}=\frac{a_{21} b_{20}}{3 a_{30}+b_{21}}+\frac{3 a_{30}}{2 b_{20}}, \quad a_{11}=\frac{2 a_{12} b_{20}}{3 a_{30}+2 b_{21}}, \\
& b_{30}=0 \text {. }
\end{aligned}
$$

In this way, System $\left(S_{2}\right)$ is compatible. We can now determine the function $f_{1}(y)$, in order to obtain an explicit expression for the constant of motion $f(x, y)$.

From Equation (3), and assuming that

$$
a_{30}\left(3 a_{30}+b_{21}\right)\left(3 a_{30}+2 b_{21}\right) \neq 0, \quad b_{21} \neq 0,
$$

we obtain

$$
\begin{aligned}
f_{1}(y)=2\left(\frac{y^{2}\left(b_{20}+b_{21} y\right)^{\frac{3 a_{30}}{b_{21}}}}{3 a_{30}}-\right. & \frac{2\left(b_{20}+b_{21} y\right)^{\frac{3 a_{30}+b_{21}}{b_{21}}}}{3 a_{30}\left(3 a_{30}+b_{21}\right)} \\
& \left.+\frac{2\left(b_{20}+b_{21} y\right)^{\frac{3 a_{30}+2 b_{21}}{b_{21}}}}{3 a_{30}\left(3 a_{30}+b_{21}\right)\left(3 a_{30}+2 b_{21}\right)}\right) .
\end{aligned}
$$

Now, from Equations $(\alpha)$ and $(\beta)$, and assuming that

$$
a_{30}\left(3 a_{30}+b_{21}\right)\left(3 a_{30}+2 b_{21}\right)\left(a_{30}+b_{21}\right) \neq 0, \quad b_{21} \neq 0,
$$

we determine $f_{2}(x, y)$ :

$$
\begin{aligned}
f_{2}(x, y)= & 2\left(\frac{x^{2}}{2}+\frac{b_{20}}{3} x^{3}+\frac{b_{11}}{2} x^{2} y+b_{02} x y^{2}+\frac{b_{21}}{3} x^{3} y+\frac{b_{12}}{2} x^{2} y^{2}+b_{03} x y^{3}\right. \\
& +2\left(\frac{y\left(b_{20}+b_{21} y\right)}{3 a_{30}}-\frac{\left(b_{20}+b_{21} y\right)^{2}}{3 a_{30}\left(3 a_{30}+b_{21}\right)}\right) \\
& +2 a_{03}\left(\frac{y^{3}\left(b_{20}+b_{21} y\right)}{3 a_{30}}-\frac{3 y^{2}\left(b_{20}+b_{21} y\right)^{2}}{3 a_{30}\left(3 a_{30}+b_{21}\right)}\right. \\
& +\frac{6 y\left(b_{20}+b_{21} y\right)^{3}}{3 a_{30}\left(3 a_{30}+b_{21}\right)\left(3 a_{30}+2 b_{21}\right)} \\
& \left.\left.-\frac{6\left(b_{20}+b_{21} y\right)^{4}}{3 a_{30}\left(3 a_{30}+b_{21}\right)\left(3 a_{30}+2 b_{21}\right)\left(3 a_{30}+3 b_{21}\right)}\right)\right)\left(b_{20}+b_{21} y\right)^{\frac{3 a_{30}-b_{21}}{b_{21}}}
\end{aligned}
$$

then, we conclude with the following theorem: 
Theorem 1. Under conditions

$b_{11}=\frac{2 a_{21} b_{20}}{3 a_{30}+b_{21}}+\frac{b_{21}}{b_{20}}, \quad b_{02}=\frac{a_{12} b_{20}}{3 a_{30}+2 b_{21}}, \quad b_{12}=\frac{2 a_{21} b_{21}}{3 a_{30}+b_{21}}$

$b_{03}=\frac{a_{12} b_{21}}{3 a_{30}+2 b_{21}}, \quad a_{20}=\frac{a_{21} b_{20}}{3 a_{30}+b_{21}}+\frac{3 a_{30}}{2 b_{20}}, \quad a_{11}=\frac{2 a_{12} b_{20}}{3 a_{30}+2 b_{21}}$,

$b_{30}=0$,

with $b_{20} \neq 0, b_{21} \neq 0, a_{30}\left(3 a_{30}+b_{21}\right)\left(3 a_{30}+2 b_{21}\right)\left(a_{30}+b_{21}\right) \neq 0$, the origin is a center of $\left(S_{1}\right)$ and the constant of motion is given by

$$
\begin{aligned}
f(x, y)= & \left(2\left(\frac{y\left(b_{20}+b_{21} y\right)}{3 a_{30}}-\frac{\left(b_{20}+b_{21} y\right)^{2}}{3 a_{30}\left(3 a_{30}+b_{21}\right)}\right)\right. \\
& +2 a_{02}\left(\frac{y^{2}\left(b_{20}+b_{21} y\right)}{3 a_{30}}-\frac{2 y\left(b_{20}+b_{21} y\right)^{2}}{3 a_{30}\left(3 a_{30}+b_{21}\right)}\right. \\
& \left.+\frac{2\left(b_{20}+b_{21} y\right)^{3}}{3 a_{30}\left(3 a_{30}+b_{21}\right)\left(3 a_{30}+2 b_{21}\right)}\right) \\
& +2 a_{03}\left(\frac{y^{3}\left(b_{20}+b_{21} y\right)}{3 a_{30}}-\frac{3 y^{2}\left(b_{20}+b_{21} y\right)^{2}}{3 a_{30}\left(3 a_{30}+b_{21}\right)}\right. \\
& +\frac{6 y\left(b_{20}+b_{21} y\right)^{3}}{3 a_{30}\left(3 a_{30}+b_{21}\right)\left(3 a_{30}+2 b_{21}\right)} \\
& \left.-\frac{6\left(b_{20}+b_{21} y\right)^{4}}{3 a_{30}\left(3 a_{30}+b_{21}\right)\left(3 a_{30}+2 b_{21}\right)\left(3 a_{30}+3 b_{21}\right)}\right) \\
& +x^{2}+\frac{2}{3} b_{20} x^{3}+b_{11} x^{2} y+2 b_{02} x y^{2} \\
& \left.+\frac{2}{3} b_{21} x^{3} y+b_{12} x^{2} y^{2}+2 b_{03} x y^{3}\right)\left(b_{20}+b_{21} y\right)^{\frac{3 a_{30}-b_{21}}{b_{21}}} .
\end{aligned}
$$

We have written the constant of motion in such a way that it will be possible later on, to guess its general form for polynomial systems of arbitrary degree.

Let us remark that all the constants of motion that we have found in this section are also linear in the coefficient $a_{03}$.

New integrability cases and new constants of motion can be obtained from the above results by interchanging $x$ and $y, a_{i j}$ and $b_{j i}$. This is due to the invariance of $(S)$ under the transformation $x \leftrightarrow y, a_{i j} \leftrightarrow b_{j i}$, $t \rightarrow-t$.

\section{General case}

Using the results obtained in Section 1, we remark that when we seek for a constant of motion which is linear in a coefficient of $y^{m}$ in $P(x, y)$, 
with $1 \leq m \leq 3$, we obtain one which is linear in all coefficients of $y^{m}$. It is natural to think that this result will be valid for polynomial systems of an arbitrary degree $n$. In order to verify this hypothesis, we have carried out similar calculations as in Section 1, for polynomial systems up to degree 8. We have found analogous results as for the case $n=3$ : in all the cases, the constant of motion is linear in all coefficients of $y^{m}$ in $P(x, y)$. In addition, it is always possible to find explicit expressions of the constant of motion.

By throughly inspecting expressions for integrability conditions and constants of motion up to degree 8 , we have been able to induce analogous results for polynomial systems of an arbitrary degree. Let us remark that, up to $n=8$, we have found that for the System $(S)$, which has $(n-1)(n+4)$ coefficients, the number of integrability conditions is given by $\frac{(n-1)(n+4)}{2}$ for the principal case, where $\prod_{k=0}^{n}\left(n a_{n, 0}+k b_{n-1,1}\right) \neq 0$, $b_{n-1,0} \neq 0$ and $b_{n-1,1} \neq 0$.

The above remarks lead us to conjecture the following theorem:

Theorem 2.1. Consider the System $(S)$. Assuming that

$$
\prod_{r=0}^{n}\left(n a_{n, 0}+r b_{n-1,1}\right) \neq 0, \quad b_{n-1,0} \neq 0, \quad b_{n-1,1} \neq 0
$$

and $a_{i j}=0$ if $i+j>n$ or $j<0$, and under conditions

$$
\begin{aligned}
a_{20}= & \frac{n a_{n, 0}}{2 b_{n-1,0}}+\sum_{m \in^{*}} \frac{(-1)^{m+1} m ! a_{2, m} b_{n-1,0}^{m}}{\prod_{r=1}^{m}\left(n a_{n, 0}+r b_{n-1,1}\right)} \\
b_{11}= & \frac{b_{n-1,1}}{b_{n-1,0}}+2 \sum_{m \in^{*}} \frac{(-1)^{m+1} m ! a_{2, m} b_{n-1,0}^{m}}{\prod_{r=1}^{m}\left(n a_{n, 0}+r b_{n-1,1}\right)}, \\
a_{11}= & \sum_{m \in^{*}} \frac{(-1)^{m+1}(m+1) ! a_{1,1+m} b_{n-1,0}^{m}}{\prod_{r=1}^{m}\left(n a_{n, 0}+(r+1) b_{n-1,1}\right)} \\
b_{02}= & \frac{1}{2} \sum_{m \in *} \frac{(-1)^{m+1}(m+1) ! a_{1,1+m} b_{n-1,0}^{m}}{\prod_{r=1}^{m}\left(n a_{n, 0}+(r+1) b_{n-1,1}\right)} \\
b_{i, j}= & (i+1)\left(\frac{a_{i+1, j-1} b_{n-1,1}}{n a_{n, 0}+(j-1) b_{n-1,1}}+\left(n a_{n, 0}-b_{n-1,1}\right) \sum_{m \in *}(-1)^{m-1}\right. \\
& \left.\frac{(j+m-1) !}{j !} \quad \frac{a_{i+1, m+j-1} b_{n-1,0}^{m}}{\prod_{r=j}^{m+j}\left(n a_{n, 0}+(r-1) b_{n-1,1}\right)}\right) \\
& 2 \leq i+j \leq n, \quad(i, j) \neq(0,2), \quad(i, j) \neq(1,1),
\end{aligned}
$$


$O(0,0)$ is a center and the associated constant of motion is

$$
\begin{aligned}
f(x, y)=\sum_{m=0}^{n} a_{0, m} & \sum_{l=0}^{m}(-1)^{l} \frac{m !}{(m-l) !} \\
& \frac{y^{m+1-l}\left(b_{n-1,0}+b_{n-1,1} y\right)^{\frac{n a_{n, 0}+l b_{n-1,1}}{b_{n-1,1}}}}{\prod_{k=0}^{l}\left(n a_{n, 0}+k b_{n-1,1}\right)}+h(x, y),
\end{aligned}
$$

with

$$
h(x, y)=\left(\sum_{j=0}^{n} \sum_{i=0}^{j} b_{i, j-i} \frac{x^{i+1}}{i+1} y^{j-i}\right)\left(b_{n-1,0}+b_{n-1,1} y\right)^{\frac{n a_{n, 0}-b_{n-1,1}}{b_{n-1,1}}}
$$

and $a_{0,0}=b_{0,0}=a_{1,0}=b_{0,1}=0, a_{0,1}=b_{1,0}=1$.

We recall that this conjectured theorem has been verified for $2 \leq n \leq$ 8. Let us remark that we have also considered all the particular cases by using the same method. We have found nine particular cases, but due to the size of the calculations we have not included these cases in the present paper. We have also seek constants of motion which are linear in the remaining coefficients of $\left(S_{1}\right)$, but the resulting differential equations for the functions $f_{1}(x, y)$ and $f_{2}(x, y)$ are in all cases incompatible.

Much of calculations performed in this work have been made with Mathematica computer algebra system.

\section{References}

1. D. Schlomiuk, J. Guckenheimer And R. RAnd, Integrability of plane quadratic vector field, Exp. Math 8 (1990), 3-25.

2. D. Schlomiuk, Algebraic particular integrals, integrability and the problem of the center, Trans. Amer. Math. Soc. 338 (1993), p. 799.

3. H. ŻOLADEK, Quadratic systems with center and their perturbations, J. Differential Equations 109 (1994), 223-273.

4. V. A. Lunkevich and S. K. Sibirski, On the condition for a center, Differential'nye Uravneniyad 1 (1965), 53-66.

5. J. Chavarriga, Integrable systems in the plane with center type linear part, Applicationes Mathematicae 22 (1994), 285-309.

6. I. S. KuKLES, Necessary and sufficient conditions for the existence of a center, Dokl. Akad. Nauk. 42 (1944), 160-163. 
7. I. S. KukLes, Sur quelques cas de distinction entre un foyer et un centre, Dokl. Akad. Nauk, SSSR 42 (1944), 208-211.

8. X. F. Jin And D. M. WAnG, On the conditions of Kukles for the existence of a center, Bull. London Math. Soc. 22 (1990), 1-4.

9. L. A. Cherkas, Conditions for the equation $y y^{\prime}=\sum_{i=0}^{3} P_{i}(x) y^{i}$ to have a center, Differential Equations 14 (1978), 1133-1138.

10. C. J. Christopher And N. G. Lloyd, On the paper of Jin and Wang concerning the conditions for a center in certain cubic systems, Bull. London Math. Soc. 22 (1990), 5-12.

11. H. ŻolADEK, The classification of reversible cubic systems with center, Topol. Methods Nonlinear Anal. 4 (1994), 79-136.

12. J. SokUlSky, The beginning of the classification of Darboux integrals for cubic systems with center, Preprint, Warsaw, 1993.

13. J. ArGÉmi, Sur les points singuliers multiples de systèmes dynamiques dans ${ }^{2}$, Ann. Mat. Pure et Appl., Ser. IV 79 (1968), 35-70.

14. M. B. Sevryuk, "Reversible Systems," Lecture Notes in Math. $\mathbf{1 2 1 1}, 1986$.

15. G. Darboux, Mémoire sur les équations différentielles algébriques du premier ordre et du premier degré (Mélanges), Bull. des Sc. Math. (1878), 60-96, 123-144, 151-200.

16. M. J. Prelle AND M. D. Singer, Elementary first integral of differential equations, Trans. Amer. Math. Soc. 279(1) (1981), 215229.

17. D. Schlomiuk, Elementary first integrals of differential equations and invariant algebraic curves, Expositiones Mathematicae 11 (1993), 433-454.

18. D. Schlomiuk, Algebraic and geometric aspects of the theory of polynomial vector fields, in "Bifurcations and Periodic Orbits of Vector Fields," (ed. Dana Schlomiuk), Kluwer Academic Press, NATO ASI Series, Series C-408, 1993, pp. 429-467.

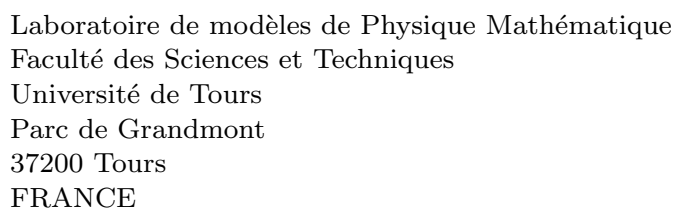

Primera versió rebuda el 23 d'Octubre de 1995, darrera versió rebuda el 10 de Novembre de 1995 